\title{
Mesoscale Organization of CuO Nanoribbons: Formation of "Dandelions"
}

\author{
Bin Liu and Hua Chun Zeng* \\ Department of Chemical and Biomolecular Engineering, Faculty of Engineering \\ National University of Singapore, 10 Kent Ridge Crescent, Singapore 119260
}

\section{SI-1. EDX analysis for CuO crystal strips}

The following EDX spectrum was measured together with HRTEM images (SI-4) for rhombic CuO crystal strips.

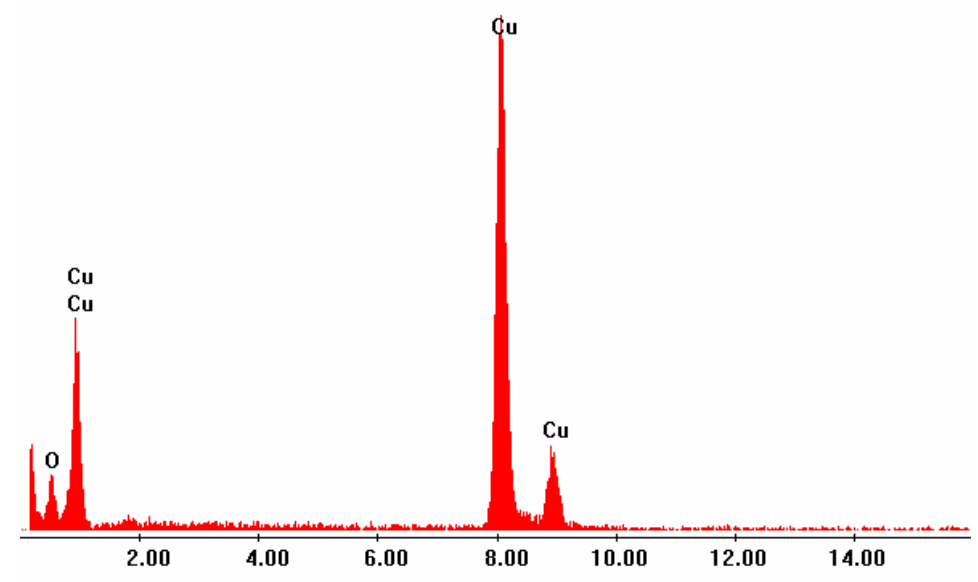

\section{SI-2. Thickness of $\mathrm{CuO}$ crystal strips and their intercrystal space (also see SI-5)}

The following FESEM image was taken perpendicularly to a $\mathrm{CuO}$ microsphere (i.e., parallel to some of the crystal strips), where the thin $\mathrm{CuO}$ crystal strips can be clearly seen and their thickness can be determined from those standing perpendicularly to the paper (in the central part) with a greater magnification (not shown). 


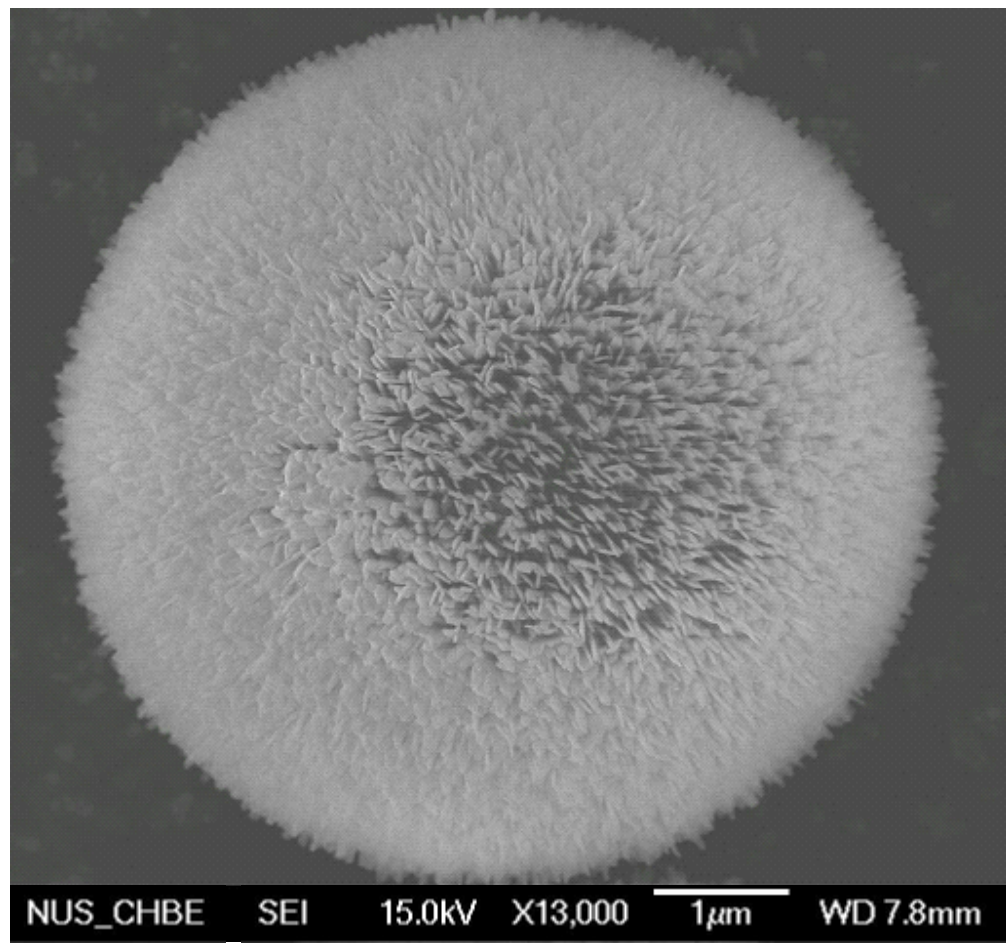

\section{SI-3. CuO microspheres synthesized at lower temperatures}

In addition to those described in the main text, the $\mathrm{CuO}$ microspheres can also be prepared at temperatures below $100^{\circ} \mathrm{C}$. Typical experiments of this type can be summarized: $1.208-2.416 \mathrm{~g}$ of $\mathrm{Cu}\left(\mathrm{NO}_{3}\right)_{2} \cdot 3 \mathrm{H}_{2} \mathrm{O}$ was dissolved in $100 \mathrm{~mL}$ of pure ethanol. Then $50-70 \mathrm{~mL}$ ammonia solution $(32 \%)$ was added to the above solution. The solution became clear and dark blue. After that, $20 \mathrm{~mL}$ of $1.0 \mathrm{M} \mathrm{NaOH}$ solution was added drop-by-drop; the solution was still kept clear. Afterwards $0-20 \mathrm{~g}$ of $\mathrm{NaNO}_{3}$ was added. The mixture was then transferred to a glass bottle and kept either in an electric oven at $60-100^{\circ} \mathrm{C}$ without stirring or inside an oil bath at $60-100^{\circ} \mathrm{C}$ with constant stirring for $24-48 \mathrm{~h}$. The products were washed several times with DI water and pure ethanol and separated by sedimentation. The washed precipitates were dried in an electric oven at $60^{\circ} \mathrm{C}$ overnight. In general, finer $\mathrm{CuO}$ crystal strips can be achieved from the lowtemperature synthesis. However, it is anticipated that the integrity of the spheres will be lower than that prepared at higher temperatures. The following are two representative SEM images of these $\mathrm{CuO}$ microspheres (synthesized at $80^{\circ} \mathrm{C}$ for $48 \mathrm{~h}$ ).
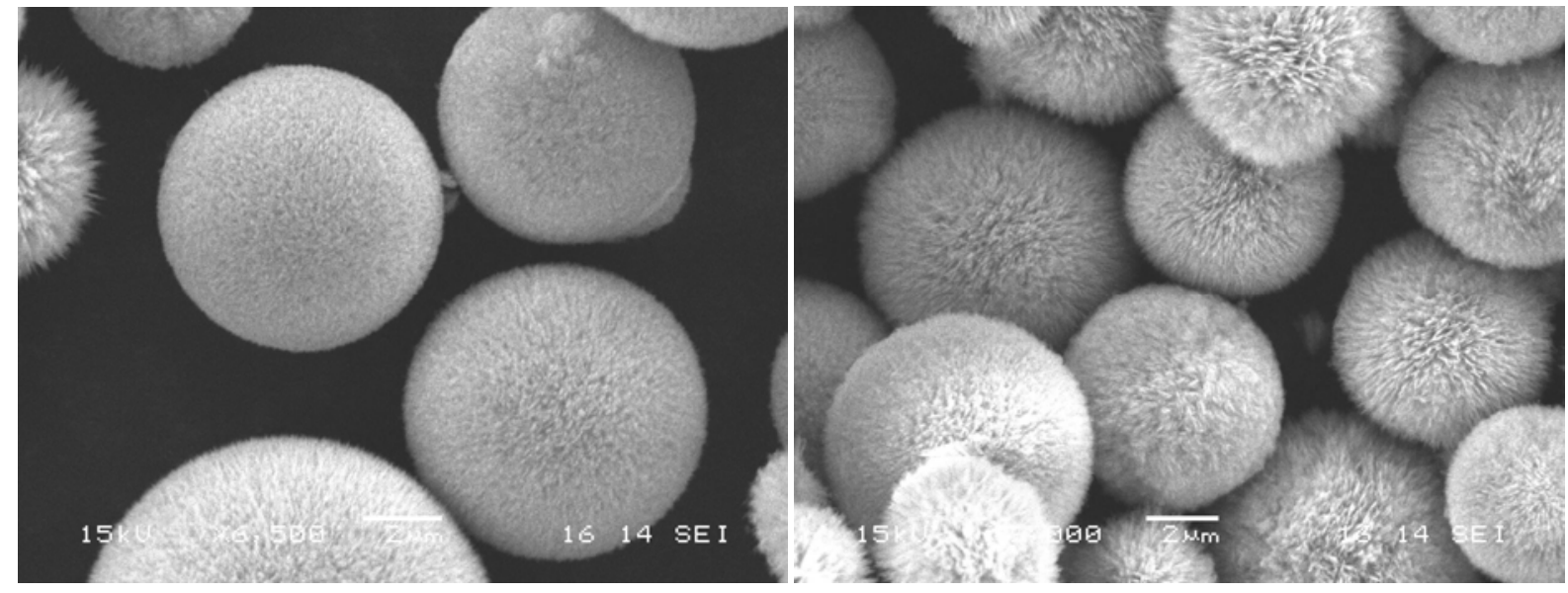


\section{SI-4. Structural details of $\mathrm{CuO}$ nanoribbons in the crystal strips}

The following two HRTEM images show (a) oriented aggregation of tiny $\mathrm{CuO}$ nanoribbons (in the tip area of a rhombic $\mathrm{CuO}$ crystal strip), and (b) $d_{110}$ lattice fringes of the attached $\mathrm{CuO}$ nanoribbons.

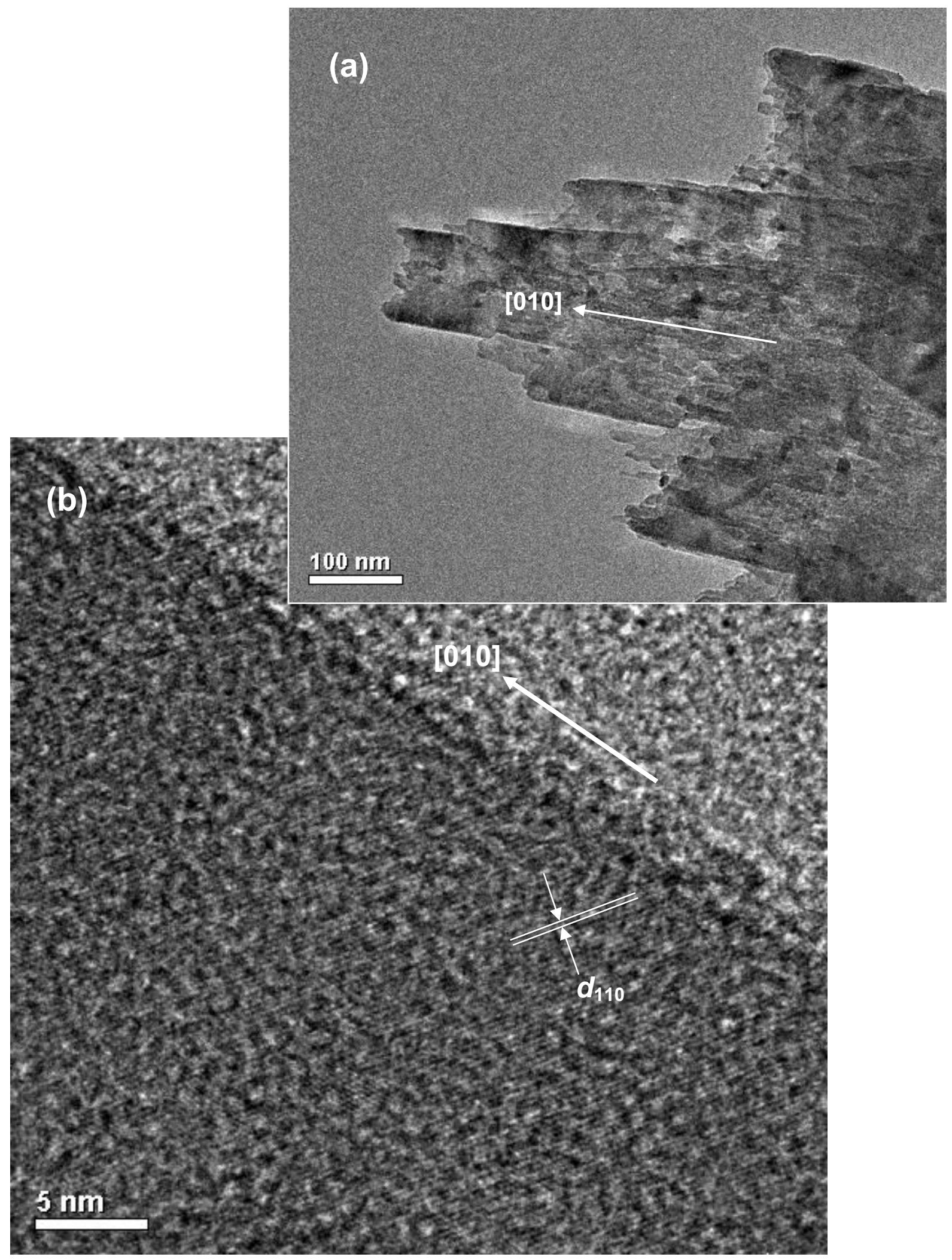


The following two TEM images (c and d) show that rhombic $\mathrm{CuO}$ crystal strips were assembled from small $\mathrm{CuO}$ nanoribbons in all our syntheses regardless of size of crystal strips.
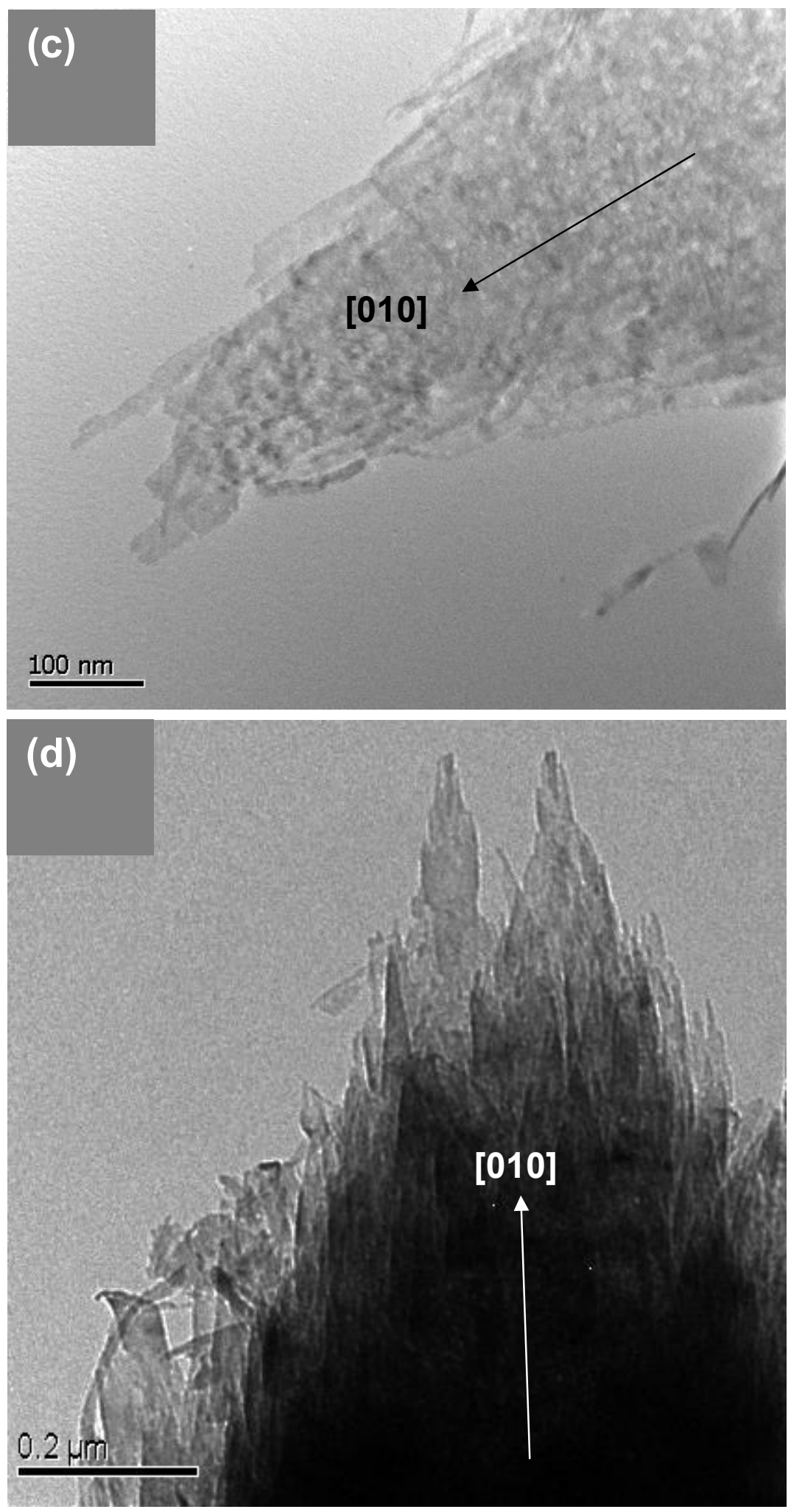


\section{SI-5. Shell wall texture and hollow interior of $\mathrm{CuO}$ microspheres}

The following FESEM image was taken for a crashed $\mathrm{CuO}$ microsphere, where the wall texture and interior structure of the concentrically assembled $\mathrm{CuO}$ crystal strips are shown. Porous and puffy nature of the microsphere can also be clearly observed.

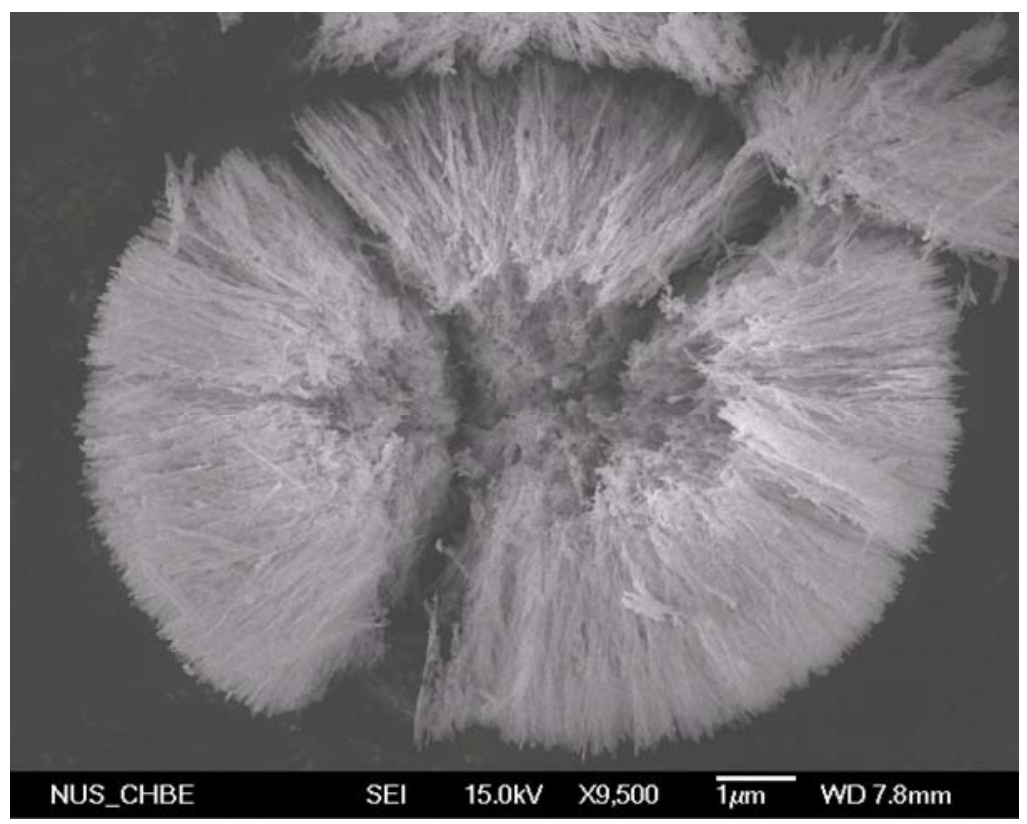

\section{SI-6. Time-dependent formation of $\mathrm{CuO}$ microspheres}

The formation mechanism of $\mathrm{CuO}$ microspheres from $\mathrm{CuO}$ crystal strips are indicated in the following series of experiments $\left(15 \mathrm{~mL}\right.$ of $32 \%$ ammonia solution was added to $25 \mathrm{~mL}$ of $1.0 \mathrm{M} \mathrm{Cu}\left(\mathrm{NO}_{3}\right)_{2}$ ethanol solution. Then $5 \mathrm{~mL}$ of $1.0 \mathrm{M} \mathrm{NaOH}$ was added. The autoclave was transferred to an electric oven at $100^{\circ} \mathrm{C}$ and kept for 1,4 , and $12 \mathrm{~h}$ respectively). It is shown that the $\mathrm{CuO}$ crystal strips were readily formed after a short reaction time (even after $1 \mathrm{~h}$ ). With a longer reaction time, the $\mathrm{CuO}$ crystal strips were gradually assembled to $\mathrm{CuO}$ microspheres $(4 \mathrm{~h}$ and $12 \mathrm{~h}$ ). Under the similar conditions, almost perfect spherical assemblies in $100 \%$ morphological yield can be obtained after $24 \mathrm{~h}$ [not shown here, see Figure $2\left(100^{\circ} \mathrm{C}\right.$ for $24 \mathrm{~h}$ ) in the main text].

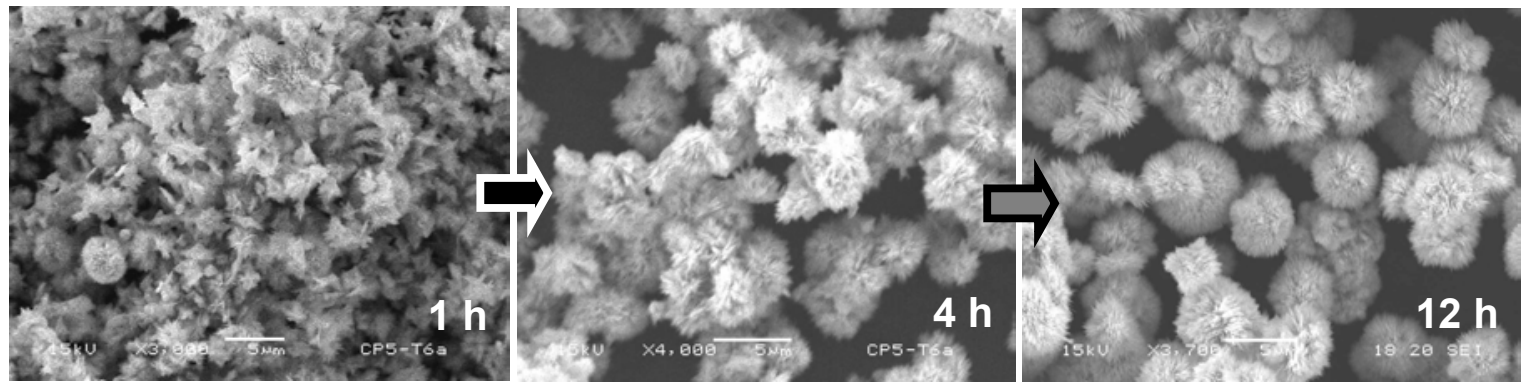




\section{SI-7. Donut-like intermediate structure prior to the formation of final $\mathrm{CuO}$ microspheres}

In addition to the results shown in SI-6, structural intermediate during the sphere formation can be arrested with larger $\mathrm{CuO}$ crystal strips that were formed at higher temperatures (see the main text). The following series of SEM images shows that prior to the formation of final $\mathrm{CuO}$ microspheres, the rhombic $\mathrm{CuO}$ crystal strips self-organized into a donut-like structure, where the $\mathrm{CuO}$ crystal strips are stacked along the equatorial rings [indicated with arrows, SEM image (b); (b) is the framed area of (a)]. Final closing (i.e., completion) of the spherical assemblies can also be visualized in the SEM images (c) and (d) (indicated with arrows). Experimental conditions: $20 \mathrm{~mL}$ of $32 \%$ ammonia solution was added to $20 \mathrm{~mL}$ of $1.0 \mathrm{M}$ $\mathrm{Cu}\left(\mathrm{NO}_{3}\right)_{2}$ ethanol solution, followed by an addition of $5 \mathrm{~mL}$ of $1.0 \mathrm{M} \mathrm{NaOH}$. This solution mixture was then transferred to a Teflon-lined autoclave and heated in an electric oven at $180^{\circ} \mathrm{C}$ for $24 \mathrm{~h}$.
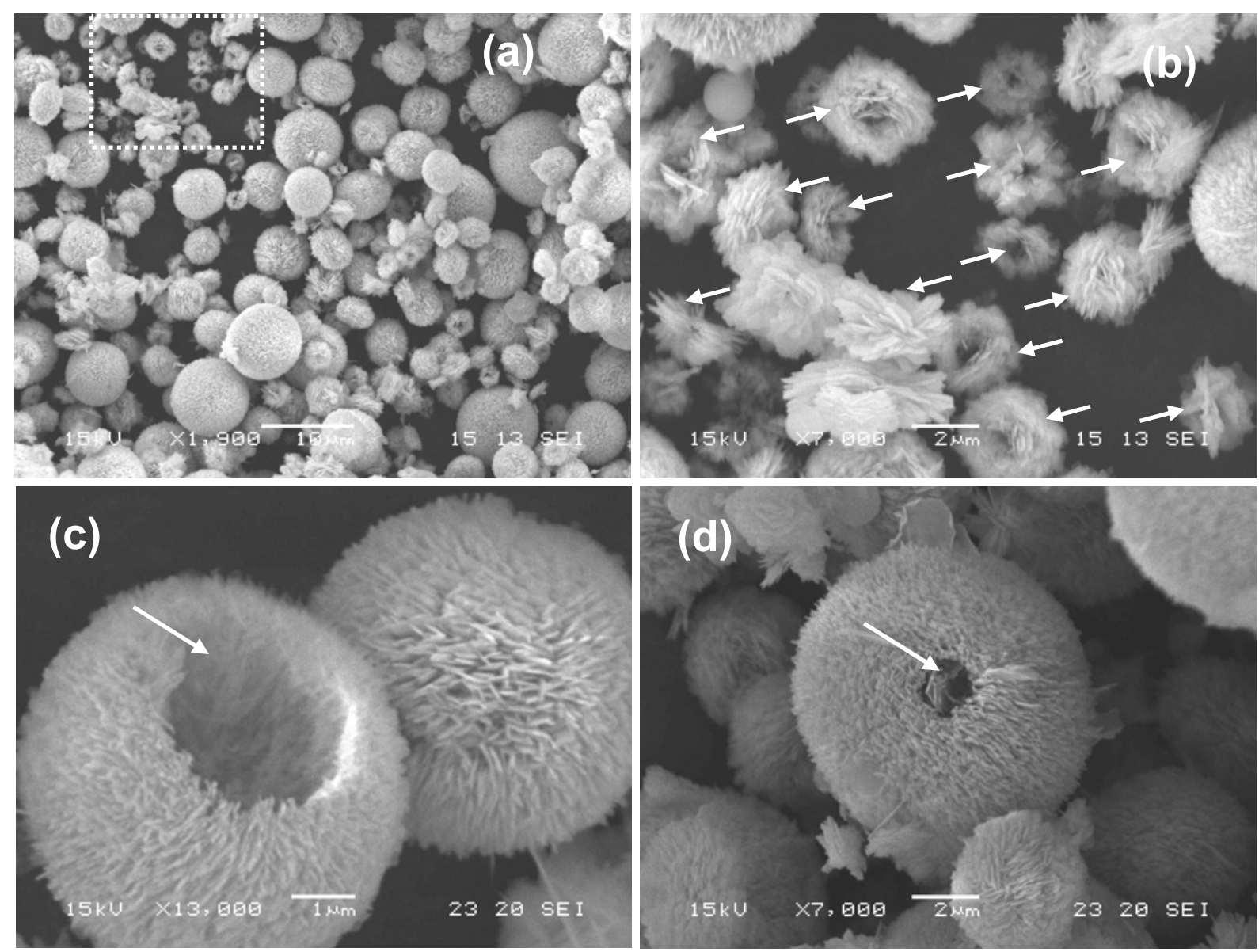


\section{SI-8. Integrity testing for $\mathrm{CuO}$ microspheres}

The interconnect bonding among $\mathrm{CuO}$ crystal strips in microspheres has been investigated with ultrasonic treatments. The following two SEM images indicate that the good interconnectivity exists among the $\mathrm{CuO}$ crystal strips, even for those synthesized at low temperatures; the sample tested here was synthesized at $80^{\circ} \mathrm{C}$ for $48 \mathrm{~h}$, and later treated with $6 \mathrm{~h}$ of sonication in an ultrasonic water bath.
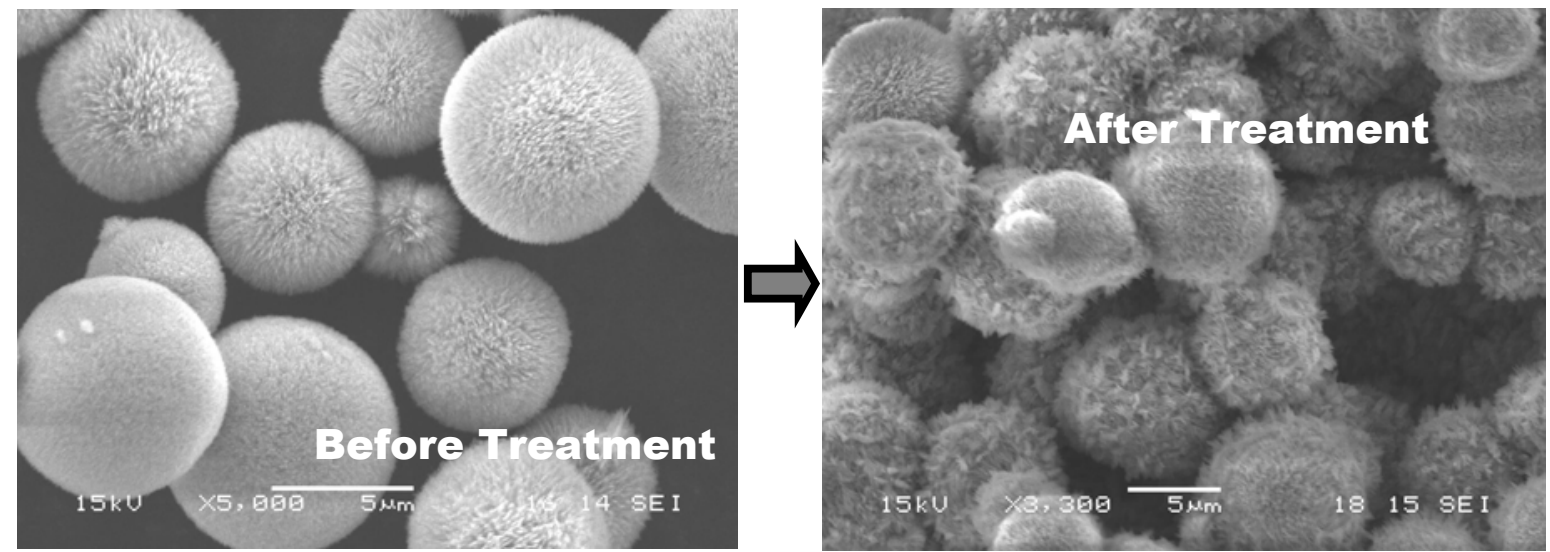

\section{SI-9. Interconnection of $\mathrm{CuO}$ microspheres (external bonding capacity)}

Some joined $\mathrm{CuO}$ microspheres can be occasionally observed in as-prepared samples, as shown in the following two SEM images (synthesized at $60^{\circ} \mathrm{C}$ for $48 \mathrm{~h}$ ). This type of interconnectivity is mainly observed for samples synthesized at temperatures below $100^{\circ} \mathrm{C}$, as a result of low fluid convection at low temperature and thus low mobility of the forming microspheres.
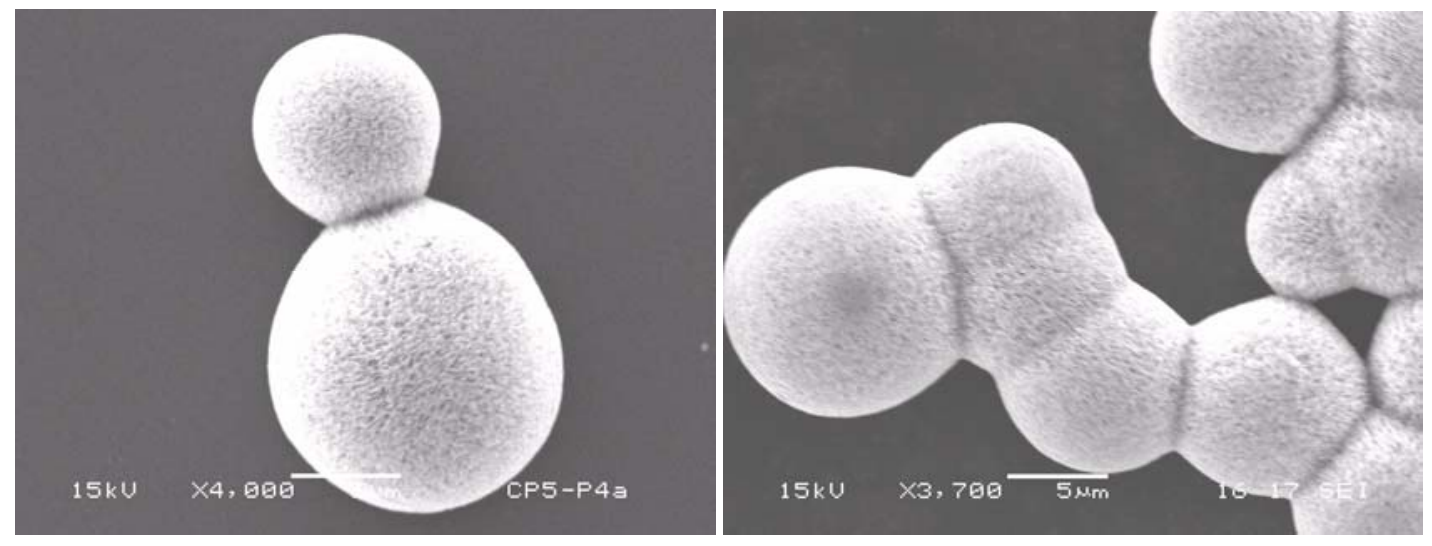